\title{
Long Duration Gamma-ray Flares and High Energy Solar Energetic Particles: Is there a Connection?
}

\author{
G.A. de Nolfo*1, A. Bruno ${ }^{1}$, J.M. Ryan ${ }^{2}$, S. Dalla ${ }^{3}$, J. Giacalone ${ }^{4}$, I.G. Richardson ${ }^{1,5}$, \\ E.R. Christian ${ }^{1}$, On Behalf of the PAMELA Collaboration \\ ${ }^{1}$ NASA Goddard Space Flight Center, Greenbelt, MD \\ E-mail: georgia.a.denolfo@nasa.gov \\ ${ }^{2}$ Space Science Center, University of New Hampshire, Durham NH, USA \\ ${ }^{3}$ Jeremiah Horrocks Institute, University of Central Lancashire, UK \\ ${ }^{4}$ Department of Planetary Sciences, University of Arizona, Tuscon, AZ, USA \\ ${ }^{5}$ Department of Astronomy, University of Maryland, College Park MD, USA
}

Long Duration Gamma-Ray Flares (LDGRFs) are characterized by delayed and long-duration gamma-ray emission above $\sim 50 \mathrm{MeV}$. Despite dozens of observations in the last decade with Fermi/LAT, the nature of this emission has been a challenge to explain. The highest energy emission has generally been attributed to the decay of pions produced by the interaction of highenergy protons with ambient solar material. The fact that the $\gamma$-ray emission is delayed from the onset of the initial eruption and that the emission is, in some cases, unusually long in duration suggests that particle acceleration occurs within large volumes extending to high altitudes, either by stochastic acceleration within large coronal loops or by back-precipitation from CME-driven shocks. We have tested these models by a making direct comparisons between the properties of the accelerated ion population at the flare derived from the observations of Fermi/LAT and those of solar energetic particles detected at Earth by PAMELA at comparable high energies. We investigated 27 high-energy gamma ray events (from [1]), and for 14 events we compare the two populations (SEPs in space and the interacting population at the Sun) and discuss the implications in terms of potential sources of the LDGRFs.

36th International Cosmic Ray Conference -ICRC2019-

July 24th - August 1st, 2019

Madison, WI, U.S.A.

${ }^{*}$ Speaker. 
Solar flares are often complex, involving photon signatures that span a broad range in energy, large and dynamic magnetic structures, coronal mass ejections (CMEs), and solar energetic particles (SEPs). While it is clear from X-ray and $\gamma$-ray observations that electrons and ions are accelerated in flares and interact with the solar atmosphere, the origin of SEPs and their relationship to flare-accelerated particles is still not understood. Particle transport complicates the interpretation of SEP observations, while the interacting flare particles can only be studied indirectly through neutral radiation. Comparisons of the two populations can help to resolve the ambiguity of their origins. Early studies took advantage of inferring the spectral shape of the interacting energetic particle population from the $\gamma$-ray line emission (see for example $[2,3,6,5,4,20,7]$ ). However, such comparisons correspond to interacting proton energies $<50 \mathrm{MeV}$. Fermi/LAT observations of the Sun have since changed our picture of high-energy $\gamma$-rays and thus the associated population of high-energy interacting particles.

Furthermore, solar flares exhibit complicated time evolution with the time profile of the electromagnetic emissions commonly divided into several phases [8]. The initial "precursor" phase typically lasts 10-30 minutes and is identified by the gradual buildup of soft X-ray and EUV emission. The "impulsive" phase that follows lasts from 10-1000 s and exhibits emission in the microwaves, hard X-rays, and $\gamma$-rays. In the third "explosive" phase, the $\mathrm{H}_{\alpha}$ flux rises to a maximum within a few minutes. The final "gradual" phase lasts between one and several hours. A small class of events have been identified based on their extended $\gamma$-ray and neutron emission, well beyond the impulsive phase, and are known as "long duration $\gamma$-ray flares" (LDGRF) [9].

LDGRFs, identified with CGRO, SMM and now Fermi, radiate almost entirely in $\gamma$-rays with energies above $\sim 30 \mathrm{MeV}[10,9,11,12,13,14,1]$. This extended emission emanates from pion decay produced by ions above $\sim 300 \mathrm{MeV}[15,14]$. The most intense and longest duration example of an LDGRF is the 2012 March 7 event, for which $>100 \mathrm{MeV}$ emission was observed for nearly twenty hours [16]. To sustain hours of high-energy emission, particle trapping and/or continuous acceleration must take place within large magnetic loops [11, 17, 18]. CMEs may also play a role $[19,21]$, through backward particle precipitation from a CME-driven shock, although several hours of extended emission would then place the CME far from the solar surface.

In seemingly all cases, LDGRFs are accompanied by SEPs. Whether these SEPs are accelerated by the same processes that produce ions responsible for the the high-energy emission or from subsequent CME-driven shocks is still not well understood. The energetic particles responsible for LDGRF emission share several characteristics with SEPs: both are dominated by ions rather than electrons and both are delayed by several minutes from the associated X-ray event $[9,22]$, suggesting a linkage. Several studies have explored potential connections between Ground Level Enhancements (GLEs) and LDGRFs with inconclusive results [23, 9, 11, 14].

What is required is a direct comparison of the particle population interacting at the Sun in the same energy interval as the highest energy SEPs. This is now possible for the first time with the Payload for Matter-Antimatter Exploration and Light Nuclei Astrophysics (PAMELA) and the accompanying $\gamma$-ray observations with Fermi/LAT. In particular, we determine the total number of $>500 \mathrm{MeV}$ protons at $1 \mathrm{AU}, N_{S E P}$, taking advantage of the PAMELA and the Solar Terrestrial Relations Observatory (STEREO) data with the aid of transport simulations, and compare it with the number of high-energy protons at the Sun, $\mathrm{N}_{L D G R F}$, as deduced from Fermi/LAT data. We present a summary of this work, which has recently been published [36]. 


\section{PAMELA and Fermi Observations}

PAMELA is a space experiment designed to measure the charged cosmic radiation (protons, electrons, their antiparticles and light nuclei) in the energy range from several tens of $\mathrm{MeV}$ up to several hundreds of $\mathrm{GeV}$. The instrument consists of a magnetic spectrometer equipped with a silicon tracking system, a time-of-flight system shielded by an anticoincidence system, an electromagnetic calorimeter and a neutron detector. The Resurs-DK1 satellite, carrying the apparatus, was launched into a semi-polar 70 inclination, elliptical $(350-610 \mathrm{~km})$ orbit on 2006 June 15. PAMELA provided comprehensive observations of the galactic, solar and magnetospheric radiation in the near-Earth environment $[24,25,26,27,28]$. The mission lifetime was extended beyond 2015, in part due to the promise of new PAMELA SEP science, such as that presented in this paper. On 2016 January 24 the spacecraft lost contact with ground stations. The PAMELA team has recently published the detailed spectra of twenty-six high-energy SEP events between 2006 December and 2014 September [29, 30]. These observations span a broad range in energy from $\sim 80 \mathrm{MeV}$ to a few $\mathrm{GeV}$, encompassing both the low energy measurements of in-situ spacecraft and the ground-based observations of the neutron monitor network. The reported spectra are consistent with diffusive shock acceleration with clear exponential roll-overs attributed to particle escape from within the shock region during acceleration. The absence of qualitative differences between the spectra of GLE and non-GLE events suggests that GLEs are not a separate class of SEP events but they rather are the extreme end of a continuous spectral distribution. The PAMELA observations have been used by [31] to calibrate the $>80 \mathrm{MeV}$ proton channels of the Energetic Proton, Electron, and Alpha Detectors (EPEADs) and the High Energy Proton and Alpha Detectors (HEPADs) onboard GOES-13 and -15, bringing the detected spectral intensities in-line with those registered by PAMELA and thus enabling more reliable measurements of SEP spectra up to $\sim 1$ $\mathrm{GeV}$ during periods when PAMELA was not acquiring data or after the mission termination [32].

Fermi/LAT is a pair-conversion telescope with sensitivity to $\gamma$-rays between $\sim 20 \mathrm{MeV}$ and $300 \mathrm{GeV}$ [33] and a duty cycle for solar events of only $\sim 15-20 \%$ due to frequent occultation of the Sun by the Earth. However, the Fermi satellite is able to perform pointed ("target-of-opportunity") observations increasing the exposure to a particular part of the sky including the Sun. Dozens of LDGRFs have been reported since the launch of the spacecraft in 2008 June $11[12,13,14,1]$. To derive the interacting proton numbers above $500 \mathrm{MeV}$, two assumptions are made: 1) the proton distribution follows a power law, and 2) the protons have an isotropic angular distribution. All events with photon energies above $100 \mathrm{MeV}$ and directions within $12^{\circ}$ of the Sun are included in the analysis. The $\gamma$-ray background from the Earth's atmosphere is reduced by restricting the allowable events to zenith angles $<100^{\circ}$. A solar flux is obtained using a "maximum likelihood" analysis that compares the likelihood obtained by fitting the data with the solar source included with the likelihood of the null hypothesis (no solar source). Details of the analysis of LAT solar flares have been published in [34]. Estimates of the number of protons inferred from the $\gamma$-ray emission used in this study rely on the observations of [1] based on a "light bucket" approach which implements a less accurate but faster algorithm to identify transient intervals of excess highenergy solar emission. It should be noted that, in contrast to the "maximum likelihood" method, the background is not fit and the exposure is calculated with an assumed spectral model.

Figure 1 displays the heliographic distribution of solar events accompanied by LDGRFs regis- 

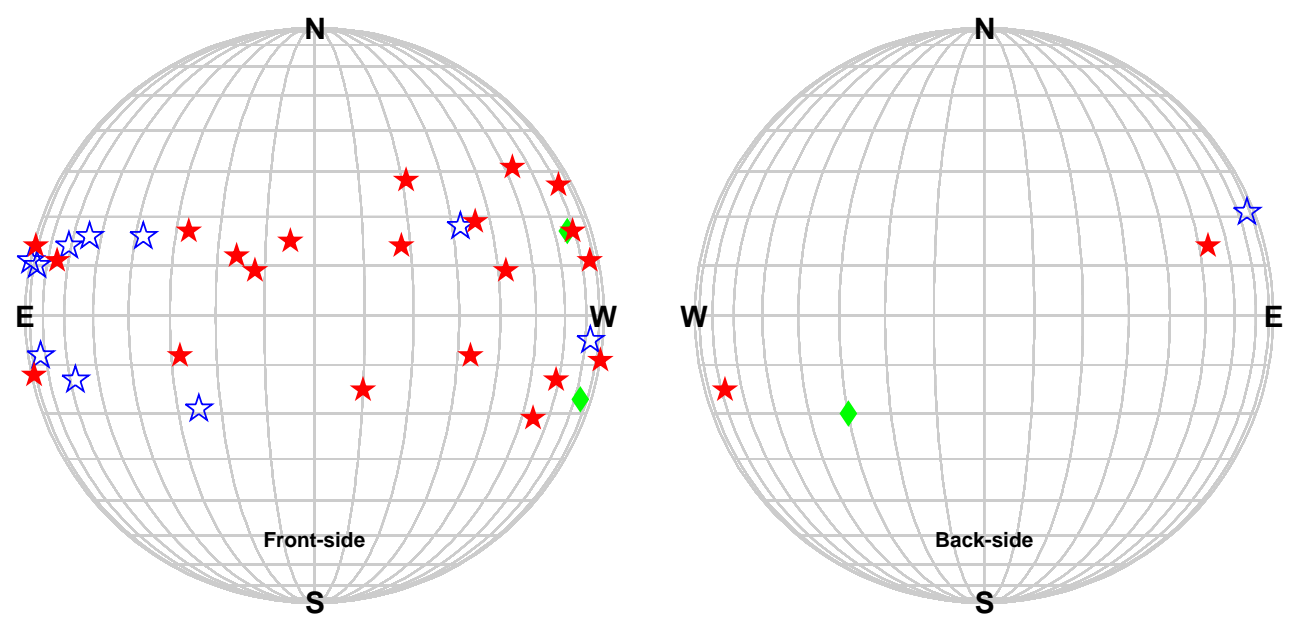

Figure 1: Heliographic distribution of solar flares associated with LDGRFs detected by Fermi/LAT and SEPs measured by near-Earth spacecraft. The red stars denote the eruptions linked to both high-energy $\gamma$-ray and SEP events, while the blue ones correspond to eruptions with no registered SEP event; for comparison, the green diamonds indicate three eruptions associated with SEP events with a statistically significant proton signal above $500 \mathrm{MeV}$ but not linked to $>100 \mathrm{MeV} \gamma$-ray emission.

\begin{tabular}{|c|c|c|c|c|c|c|c|}
\hline & \multicolumn{4}{|c|}{ SEP event } & \multicolumn{2}{|c|}{ Flare } & $\mathrm{CME}$ \\
\hline No & Onset & $>80 \mathrm{MeV}$ & $>300 \mathrm{MeV}$ & $>500 \mathrm{MeV}$ & Location & Class & Speed \\
\hline 1 & $201103 / 07,21: 30$ & $5.4 \times 10^{2, *}$ & $4.1 \times 10^{-2, *}$ & $3.4 \times 10^{-4, *}$ & N30W48 & M3.7 & 2223 \\
\hline 2 & 2011 06/07, 07:00 & $(1.5 \pm 0.1) \times 10^{5}$ & $(3.9 \pm 0.2) \times 10^{3}$ & $(4.9 \pm 0.8) \times 10^{2}$ & S21W54 & M2.5 & 1321 \\
\hline 3 & $201108 / 04,08: 05$ & $(4.9 \pm 1.4) \times 10^{4}$ & $2.5 \times 10^{2, *}$ & $1.0 \times 10^{1, *}$ & N15W39 & M9.3 & 1477 \\
\hline 4 & 2011 08/09, 08:05 & $(2.8 \pm 0.5) \times 10^{4}$ & $(5.1 \pm 2.0) \times 10^{2}$ & $(4.5 \pm 4.3) \times 10^{1}$ & N17W69 & X6.9 & 1640 \\
\hline 5 & $201109 / 06,23: 35$ & $(1.9 \pm 0.1) \times 10^{4}$ & $(7.0 \pm 1.0) \times 10^{2}$ & $(1.0 \pm 0.3) \times 10^{2}$ & N14W18 & X2.1 & 830 \\
\hline 6 & $201201 / 23,04: 20$ & $(1.5 \pm 0.2) \times 10^{5}$ & $(1.3 \pm 0.2) \times 10^{2}$ & $(6.1 \pm 3.1) \times 10^{0}$ & N28W21 & M8.8 & 2511 \\
\hline 7 & $201201 / 27,18: 40$ & $(5.6 \pm 0.4) \times 10^{5}$ & $(1.1 \pm 0.1) \times 10^{4}$ & $(2.0 \pm 0.2) \times 10^{3}$ & N27W71 & $\mathrm{X} 1.8$ & 2541 \\
\hline & & & & & N17E27 & X5.4 & 3146 \\
\hline 8 & 2012 03/07.01:40 & $(5.2 \pm 1.1) \times 10^{6}$ & $(87+3)+3$ & $(11+0.4) \times 10^{4}$ & N22E12 & $\mathrm{X} 1.3$ & 2160 \\
\hline & & & & & N16W02 & M6.3 & 1229 \\
\hline & & & & & N18W26 & M8.4 & 1638 \\
\hline 9 & 2012 05/17, 01:50 & $(2.9 \pm 0.1) \times 10^{5}$ & $(2.0 \pm 0.1) \times 10^{4}$ & $(5.3 \pm 0.4) \times 10^{3}$ & N11W76 & M5.1 & 1596 \\
\hline 10 & 2012 07/07, 00:05 & $(1.5 \pm 0.1) \times 10^{4}$ & $(2.0 \pm 0.8) \times 10^{2}$ & $(1.6 \pm 1.4) \times 10^{1}$ & S13W59 & X1.1 & 1907 \\
\hline 11 & 2013 04/11, 08:00 & $(1.0 \pm 0.1) \times 10^{5}$ & $(1.4 \pm 0.2) \times 10^{3}$ & $(8.9 \pm 3.1) \times 10^{1}$ & N09E12 & M6.5 & 1369 \\
\hline 12 & $201310 / 28,17: 55$ & $(1.7 \pm 0.1) \times 10^{4}$ & $(5.4 \pm 0.9) \times 10^{2}$ & $(7.4 \pm 2.5) \times 10^{1}$ & S06E28 & M4.4 & 1098 \\
\hline 13 & 2014 02/25, 03:00 & $(1.3 \pm 0.1) \times 10^{5}$ & $(4.2 \pm 0.2) \times 10^{3}$ & $(8.1 \pm 1.0) \times 10^{2}$ & S12E82 & $\mathrm{X} 5.0$ & 2153 \\
\hline 14 & 2014 09/01, 17:00 & $(2.1 \pm 0.1) \times 10^{5}$ & $(9.7 \pm 0.7) \times 10^{3}$ & $(1.5 \pm 0.6) \times 10^{3}$ & N14E127 & $\sim X 2.4$ & 2017 \\
\hline
\end{tabular}

Table 1: List of SEP events with a proton signal in excess of $500 \mathrm{MeV}$, associated with LDGRF detected by Fermi/LAT above $100 \mathrm{MeV}$ between 2008 August and 2014 September. The first five columns report the SEP event number, onset time (UT) and the event-integrated intensities $\left(\mathrm{sr}^{-1} \mathrm{~cm}^{-2}\right)$ above 80, 300 and 500 $\mathrm{MeV}$. For the four eruptions occurring on 2012 March 7 (no. 8), a single SEP intensity value is provided. The remaining three columns show the parent flare location and class along with the linked CME space speed (from the SOHO/LASCO catalog, https: / / cdaw.gsfc.nasa.gov/CME_list/halo/). 
tered by Fermi/LAT. Seventeen events (red stars) were found to be in common between PAMELA and Fermi/LAT, but only fourteen of them were associated with SEP events with a statistically significant proton signal in excess of $500 \mathrm{MeV}$ (see Table 1). These events are associated with $\geq$ M-class flares with hard X-ray emission extending above $100 \mathrm{keV}$ [1], and with full halo CMEs in the CDAW catalog. In addition, these events are linked to long-duration type-II and type-III radio bursts, indicating the presence of a shock and of open field lines, respectively. In particular, the measured type-II emission ranges from metric to decameter-hectometric wavelengths for most events, suggesting that the shocks accelerating particles formed close to the Sun [35]. Three eruptions (green diamonds) with $>500 \mathrm{MeV}$ protons in SEPs - including a backside eruption - are not associated with high-energy $\gamma$-ray emission, possibly due to the limited duty cycle of Fermi/LAT, while there are many more LDGRFs (blue stars) that are not associated with high-energy SEPs, most resulting from poor connectivity.

\section{Comparing Total Proton Numbers from SEPs and Interacting Protons at the Sun}

The total number of protons escaping the Sun as SEPs is determined by estimating the spatial distribution at $1 \mathrm{AU}$ and the mean free path during transport. Details of the calculations can be found in [36]. The spatial distribution is determined using multi-point observations of SEPs from PAMELA/GOES and STEREO A/B. Intensities are determined for fluences $>80 \mathrm{MeV}$, corresponding to the PAMELA threshold. Since we expect the distributions to be narrower at higher energies ( $>500 \mathrm{MeV}$ ) from GLE observations, we compute upper limits derived from spatial distributions determined above $80 \mathrm{MeV}$. While the spatial distributions are affected by several factors particularly relating to contributions from preceding events and particles being over-counted because of multiple crossings of $1 \mathrm{AU}$ due to scattering, the distributions are well described by a periodic Gaussian function with average $\sigma$ of $\sim 41^{\circ}$. Comparison of the decay times of the time-intensity profiles with test particle models of diffusion for the 2012 May 17 SEP event suggests a mean free path at $1 \mathrm{AU}$ in the range $\lambda_{0}=0.1-0.3 \mathrm{AU}$. Given that our goal is to compute upper limits for the proton numbers in space, we conservatively assume $\lambda_{0}=0.5 \mathrm{AU}$, corresponding to $>10$ crossings at $1 \mathrm{AU}$.

\section{Results \& Discussion}

The comparison between proton numbers derived from the $>100 \mathrm{MeV} \gamma$-ray emission $\left(N_{L D G R F}\right)$ and SEPs at $1 \mathrm{AU}\left(N_{S E P}\right)$ is shown in Figure 2 (see also [36]). The color code indicates a precipitation fraction (assuming SEPs are back-precipitating from the CME-driven shock), defined as $N_{L D G R F} /\left(N_{L D G R F}+N_{S E P}\right)$. The solid and the dashed curves mark the one-to-one and the one-tohundred correspondences, respectively. Fermi/LAT proton numbers are from [1], and are corrected for anisotropic effects characterized by the downward proton distribution [18]. The vertical error bars include the uncertainties on LDGRF proton numbers from [1]. For the quantity $N_{S E P}$, we show upper limits accounting for the assumptions made in the spatial distribution estimate, as discussed earlier. The $N_{S E P} / N_{L D G R F}$ ratio exhibits a large scatter, spanning more than five orders of magnitude from $\sim 7.8 \times 10^{-4}$ to $\sim 5.0 \times 10^{2}$, with a mean value of $\sim 78$. 


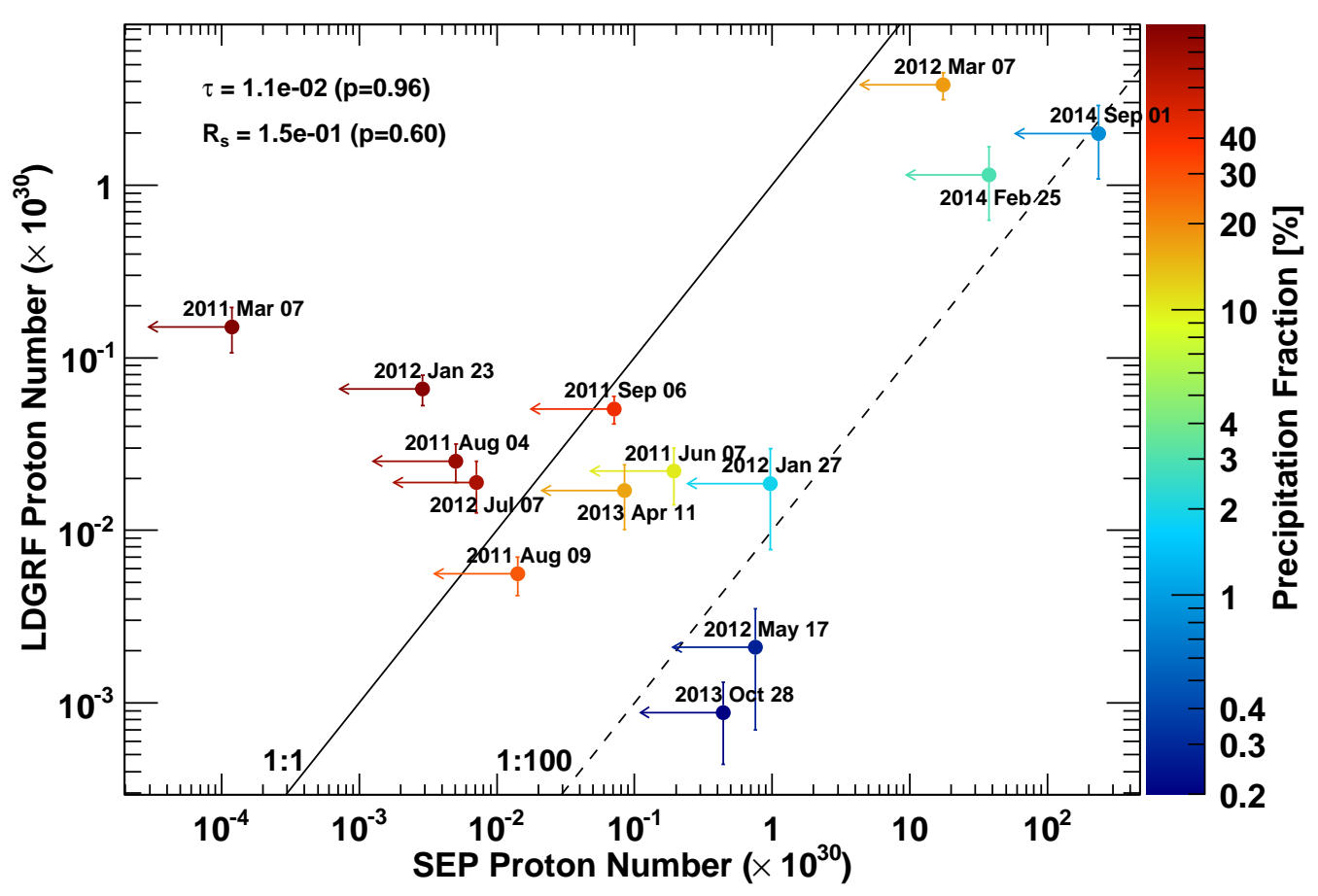

Figure 2: Number of protons deduced from Fermi/LAT [1] compared with number of protons determined from PAMELA and STEREO-A/B. The color code shows the precipitation fraction $N_{L D G R F} /\left(N_{L D G R F}+\right.$ $\left.N_{S E P}\right)$. The solid and the dashed lines mark the 1-to-1 and the 1-to-100 correspondences, respectively. The Kendall's $\tau$ and the Spearman rank $\left(R_{S}\right)$ correlation coefficients, along with their $p$-values, are also reported.

The scatter in the $N_{S E P} / N_{L D G R F}$ ratio can be explained in the context of the CME model for continuous back precipitation of energetic protons in which the magnetic connection is likely sporadic and unpredictable between the shock front, in particular the nose where acceleration is expected to be most efficient, and the Sun. However, in such a scenario one would expect the intensity-time profile in any given LDGRF to be erractic, atypical of the smooth profiles of most well-measured LDGRFs observed by Fermi/LAT. Even more problematic are the events (in the left of Figure 2) where the particle number at the flare exceeds, in some cases considerably, the particle number in space. If the particles above $500 \mathrm{MeV}$ in space were from the same population as those responsible for the $\gamma$-ray emission at the Sun, it would imply that in some cases, more than $\sim 80 \%$ of this population must be extracted from the acceleration process to produce the radiation. This loss from the shock acceleration process is in addition to other processes that reduce shock efficiency such as the finite extent of the shock and proximity to the strong magnetic fields close to the Sun. Finally, two Fermi/LAT detections on 2012 October 23 and 2012 November 27, which were not linked to CME eruptions, suggest that a fast CME is not a necessary requirement for LDGRFs.

An alternate to back precipitation is the scenario where particles are continuously accelerated within extended coronal loops and diffuse to the denser photosphere [17]. Large quasi-static loops have been associated with LDGRF emission [37, 38]. The typically smooth exponential decay of LDGRF emission is also consistent with coronal trapping, with spatial and momentum diffusion governing the precipitation of high-energy particles [39]. To accelerate protons, magnetic turbu- 
lence or Alfvén waves with $\delta \mathrm{B} / \mathrm{B}$ only $\sim 10 \%$ is necessary [40]. New radio observations can help place constraints on coronal loop size and the ambient conditions within the loop that will improve the modeling of LDRGFs within the continuous acceleration and trapping scenario (see [41]; [40]; [39]).

\section{SUMMARY}

Direct comparison of the number of interacting protons at the Sun during LDGRFs and the number of SEP protons in the energy range above the $\sim 300 \mathrm{MeV}$ pion-production threshold provides valuable insight into the origin of the $\gamma$-ray emission. An important factor in the analysis is obtaining SEP energy spectra up to a few GeV [29] that lead to estimates of the total number of $>500 \mathrm{MeV}$ protons at $1 \mathrm{AU}$, by combining PAMELA and STEREO data with the aid of transport simulations, that can be compared with the number of high-energy protons at the Sun deduced from Fermi/LAT data [1]. The results of our analysis show that the two proton numbers are uncorrelated such that their ratio spans more than five orders of magnitude. The lack of correlation, and several extreme cases where the number of protons required to account for LDGRFs far exceeds the number of SEP protons, suggests that the LDGRF emission is probably not due to the back precipitation of particles accelerated at CME-driven shocks. Moreover, two LDGRF events (2012 October 23 and November 27) demonstrate that an association with fast CMEs does not appear to be a necessary requirement for high-energy $\gamma$-ray emission. In addition, while bright flares and impulsive $>100 \mathrm{keV}$ hard X-rays are not sufficient conditions for LDGRFs, there are also several counterexamples of fast halo CMEs that were not accompanied by $>100 \mathrm{MeV} \gamma$-ray emission, though the limited exposure of the LAT instrument complicates this interpretation. An alternative explanation for LDGRFs based on continuous particle acceleration and trapping within large coronal structures that are not causally connected to the CME shock is suggested, and new remote observations of these loops [38] may help to constrain the role of such acceleration in producing LDGRF emission.

\section{References}

[1] G.H. Share, R.J. Murphy, A.K. Tolbert, et al. 2018, ApJ, 869, 182

[2] F.G. MacDonald, et al. 1985, In Proc. 17th Int. Cosmic Ray Conf., 3, 28

[3] T.T. von Rosenvinge, et al. 1981, ApJ, 290, L67

[4] R. Ramaty, et al. 1992, NASCP, 313, 480R

[5] X.M. Hua, et al., Solar Physics, 107, 351

[6] R.J. Murphy, et al. 1984, Adv. Space Res., 4, 7, 127

[7] L.I. Miroshnichenko, et al., 2003, ISCS 2003 Symposium, Slovakia, ESA SP-353

[8] Z. Svestka, 1976, Solar Flares, Vol. 8 (Dordrecht: D. Reidel: Dordrech

[9] J.M. Ryan 2000, Space Sci. Rev. , 93, 581

[10] D.J. Forrest, W.T. Vestrand, E.L. Chupp, et al. 1985, In: Proc. 19th Int. Cosmic Ray Conf., 4, 146

[11] E.L. Chupp \& J.M. Ryan 2009, Res. Astron. Astrophys., 9, 11 
[12] M. Ackermann, M. Ajello, A. Allafort, et al. 2012, ApJ, 745, 144

[13] M. Ackermann, M. Ajello, A. Allafort, et al. 2014, ApJ, 787, 15

[14] M. Ackermann, A. Allafort, L. Baldini, et al. 2017, ApJ, 835, 219

[15] G. Rank, J.M. Ryan, H. Debrunner, et al. 2001, A\&A, 378, 1046

[16] M.A.A. Ajello, A. Allafort, L. Baldini, L., et al. 2014, ApJ, 789, 20.

[17] J.M. Ryan \& M.A. Lee 1991, ApJ, 368, 316

[18] N. Mandzhavidze \& R. Ramaty 1992, ApJ, 389, 739

[19] E.W. Cliver, S.W. Kahler, W.T. Vestrand 1993, In Proc. 23rd Int. Cosmic Ray Conf., Invited, Rapporteur, and Highlight Papers, p. 91.

[20] E.W. Cliver, et al., 1993, In Proc. 23rd Int. Cosmic Ray Conf., Vol. 10, 342C

[21] L. Kocharov, T. Laitinen, R. Vainio, et al. 2015, ApJ, 806, 80

[22] S.W. Kahler, N.R. Sheeley Jr, R.A. Howard et al. 1984, J. Geophys. Res., 89, 9683

[23] R. Ramaty \& R. Murphy 1987, Space Sci. Rev. , 45, 213

[24] O. Adriani, G.C. Barbarino, G.A. Bazilevskaya, et al. 2011, ApJ, 742, 102

[25] O. Adriani, G.C. Barbarino, G.A. Bazilevskaya, et al. 2014, Physics Reports, 544, 4, 323

[26] O. Adriani, G.C. Barbarino, G.A. Bazilevskaya, et al. 2017, Il Nuovo Cimento, 10, 473

[27] A. Bruno, O. Adriani, G.C. Barbarino, et al. 2016, Adv. Space Res., 60, 788

[28] A. Bruno, O. Adriani, G.C. Barbarino, et al. 2016, J. Phys.: Conf. Ser., 675, 032006

[29] A. Bruno, G.A. Bazilevskaya, M. Boezio, et al. 2018, ApJ, 862, 97

[30] A. Bruno, E.R. Christian, G.A. de Nolfo, et al. 2019, In Proc. of 36th Int. Cosmic Ray Conf., PoS(ICRC2019)1061

[31] A. Bruno 2017, Space Weather, 15, 1191

[32] A. Bruno, E.R. Christian, G.A. de Nolfo, et al. 2019, Space Weather, 17, 419

[33] W.B. Atwood, A.A. Abdo, M. Ackermann, et al. 2009, ApJ, 697, 1071

[34] M. Ackermann, M., Ajello, A. Albert, et al. 2013, ApJ, 771, 57

[35] N. Gopalswamy, P. Mäkelä, S. Yashiro, et al. 2017, J. Phys.: Conf. Ser., 900, 012009

[36] G.A. de Nolfo, A. Bruno, J.M. Ryan, et al., 2019, ApJ, 879,90

[37] Grechnev, V. V., Kiselev, V. I., Kashapova, L. K., et al., 2018, Sol. Phys., 293, 133

[38] D.E. Gary, B. Chen, B.R. Dennis, et al. 2018, ApJ, 863, 83

[39] J.M. Ryan, G.A. de Nolfo, D.E. Gary, 2018, 42nd COSPAR Scientific Assembly, Pasadena, CA, 2018, Abstract id: D2.1-20-18

[40] J.M. Ryan, G.A. de Nolfo, A. Bruno, et al. 2018, Solar Heliospheric \& Interplanetary Environment (SHINE) conference, July 30th - August 3rd, 2018, Cocoa Beach (FL)

[41] J.M. Ryan, et al. 2019, In Proc. of 36th Int. Cosmic Ray Conf., PoS(ICRC2019)1144 